\title{
ORIGINAL ARTICLE \\ Single locus sex determination and female heterogamety in the basket willow (Salix viminalis L.)
}

\author{
P Pucholt ${ }^{1}$, A-C Rönnberg-Wästljung ${ }^{1}$ and S Berlin
}

Most eukaryotes reproduce sexually and a wealth of different sex determination mechanisms have evolved in this lineage. Dioecy or separate sexes are rare among flowering plants but have repeatedly evolved from hermaphroditic ancestors possibly involving male or female sterility mutations. Willows (Salix spp.) and poplars (Populus spp.) are predominantly dioecious and are members of the Salicaceae family. All studied poplars have sex determination loci on chromosome XIX, however, the position differs among species and both male and female heterogametic system exists. In contrast to the situation in poplars, knowledge of sex determination mechanisms in willows is sparse. In the present study, we have for the first time positioned the sex determination locus on chromosome XV in S. viminalis using quantitative trait locus mapping. All female offspring carried a maternally inherited haplotype, suggesting a system of female heterogamety or ZW. We used a comparative mapping approach and compared the positions of the markers between the $S$. viminalis linkage map and the physical maps of S. purpurea, S. suchowensis and $P$. trichocarpa. As we found no evidence for chromosomal rearrangements between chromosome XV and XIX between $S$. viminalis and $P$. trichocarpa, it shows that the sex determination loci in the willow and the poplar most likely do not share a common origin and has thus evolved separately. This demonstrates that sex determination mechanisms in the Salicaceae family have a high turnover rate and as such it is excellent for studies of evolutionary processes involved in sex chromosome turnover.

Heredity (2015) 114, 575-583; doi:10.1038/hdy.2014.125; published online 4 February 2015

\section{INTRODUCTION}

Sexual reproduction is the most common mode of reproduction among eukaryotes and results in mixing and reshuffling of alleles of the maternal and paternal genomes, yielding novel allelic combinations in future generations. In most animals, sexual reproduction has led to the evolution of separate sexes with male and female sexual organs on different individuals. In contrast, in flowering plants, only about $5 \%$ are dioecious and have male and female flowers on separate individuals (Charlesworth, 2002; Renner, 2014). Instead, the great majority, or about $90 \%$ are hermaphrodites with male and female sexual organs on the same flower, while another $5 \%$ are monoecious (Charlesworth, 2002), having separate male and female flowers on one individual. The fact that dioecy is so rare and that it has a scattered taxonomic distribution suggests that it is a derived trait and that hermaphroditism is the ancestral angiosperm state (Charlesworth, 2002). When dioecy evolves from hermaphroditism, two new mutations are required, that is, one male and one female sterilizing (Charlesworth et al., 2005). Selection will favour suppression of recombination between the two mutations and with time the nonrecombining region can expand as sexually antagonistic mutations accumulate near the sex determination locus (=SD locus), benefitting from linkage disequilibrium. A side effect of suppressed recombination is that selection will be inefficient, so that deleterious mutations can accumulate, which with time can result in structural changes in these chromosomal regions, which has happened with mammalian male-specific Y chromosomes (Charlesworth and Charlesworth, 2000; Bachtrog, 2008) and avian female-specific W chromosomes (Berlin and Ellegren, 2004; Hillier et al., 2004; Moghadam et al., 2012). Among many plants (Charlesworth and Mank, 2010) and lower vertebrates such as fish, amphibians and reptiles, sex chromosomes often display little differentiation, which is assumed to be the result of high turnover rates of the sex chromosomes (Perrin, 2009). This can happen if new sex determination mutations appear, replacing the old, before degeneration of the old sex chromosomes had time to happen (Perrin, 2009). High turnover rates can also result in transitions between male and female heterogametic sexual systems. Frequent sex chromosome turnover has been documented in several fish species (Mank and Avise, 2009) and different populations of an amphibian species even display alternate heterogametic systems (Miura, 2007). In plants, sex chromosome turnover has not been frequently encountered, although in the Silene plant genus, transition from male to female heterogamety did take place in the subsection Otites (Slancarova et al., 2013). Also in the Populus genus, transitions between male and female heterogamety seem to have happened, as is described below.

The Populus and the Salix genera belong to the Salicaceae plant family where most species are dioecious, thus suggesting that dioecy is old and evolved before the two genera diverged some $45 \mathrm{Mya}$ (Boucher et al., 2003; Manchester et al., 2006). Despite the fact that dioecy is old, heteromorphic sex chromosomes have not been found

Department of Plant Biology, Uppsala BioCenter, Swedish University of Agricultural Sciences and Linnean Center for Plant Biology, Uppsala, Sweden

1 These authors contributed equally to this work.

Correspondence: Dr S Berlin, Department of Plant Biology, Uppsala BioCenter, Swedish University of Agricultural Sciences and Linnean Center for Plant Biology, SLU, Box 7080, 75007 Uppsala, Sweden.

E-mail: sofia.berlin@slu.se

Received 10 July 2014; revised 17 November 2014; accepted 27 November 2014; published online 4 February 2015 
in any poplar or willow species studied so far. Instead, poplars have their SD loci on chromosome XIX, with a peritelomeric localization in P. nigra (Gaudet et al., 2008) and P. trichocarpa (Yin et al., 2008) and a pericentromeric localization in P. tremula, $P$. tremuloides (Pakull et al., 2009; Kersten et al., 2012) and P. alba (Paolucci et al., 2010). In P. trichocarpa, a 1-Mbp long, female-specific fragment with suppressed recombination is located in the sex determining region, supporting female heterogamety (Yin et al., 2008). In P. alba, sex was mapped on the female map but not on the male map, also supporting female heterogamety (Paolucci et al., 2010). In P. tremuloides, instead a 2-Mbp long male-specific region was found, supporting male heterogamety (Kersten et al., 2014). In P. nigra, sex was mapped to the male map and not to the female map, suggesting male heterogamety also in this species (Gaudet et al., 2008). Based on these observations it seems likely that different sex determination mechanisms exist in the Populus genus (Gaudet et al., 2008; Paolucci et al., 2010; Tuskan et al., 2012) with both male and female heterogamety present among closely related species, observations that support high rates of sex chromosome turnover in this plant genus.

Sex determination in willows has not been as extensively studied as in poplars, however, by investigating biased sex ratios in Salix viminalis (Subgenus: Vetrix), a polygenic model for sex determination was early on proposed (Alström-Rapaport et al., 1997) and the discovery of a RAPD marker (UBC354560) predominantly linked to females led to the hypothesis that females may possess some chromosomal fragment that is not present in males (AlströmRapaport et al., 1998). Furthermore, Semerikov et al. (2003) mapped a SD locus to one linkage group, however, which chromosome that linkage group belong to could not be determined at that time. Two SCAR markers (SCAR 354 and SCAR AE08) were developed from the original RAPD marker that were sequenced and analysed for sex linkage in a wider material of $S$. viminalis, where female linkage was confirmed for both markers (Gunter et al., 2003a). In S. eriocephala, SCAR AE08 was also significantly associated with females, whereas SCAR 354 was only marginally associated, however, the authors concluded that different willow species may share the same sex determination mechanism (Gunter et al., 2003b). In S. caprea on the other hand, no complete sex linkage was demonstrated albeit using a large number of markers, which led to the conclusion that different willow species do not share the same sex determination mechanism (Semerikov et al., 2003). Later on, SCAR 354 was sequenced in 12 different willow species and successful amplification was demonstrated only in species in the Vetrix and the Chamaetia subgenera, whereas those species in which amplification failed was in the Salix subgenus, possibly indicating that the locus is not present in this subgenus (Temmel et al., 2007). Interestingly, the SCAR 354 sequence mapped to chromosome XV in the $P$. trichocarpa genome (Temmel et al., 2007).

Given the dynamic nature of sex determination among willows and poplars, they together represent an attractive system for studies of processes involved in sex chromosome turnover. In the present study, the first objective was to identify and position loci associated with sex in $S$. viminalis by performing quantitative trait locus (QTL) mapping and to determine if the sexual system is male or female heterogametic. Sex was mapped in one mapping population, and then the interval for the major QTL was finemapped by increasing the number of genotyped individuals as well as the number of markers in the interval. The $S$. purpurea genome assembly was used to analyse the genomic content of the SD locus. The second objective was to investigate if $S$. viminalis and P. trichocarpa have homologous SD loci, possibly possessing the same sex determination mechanism.
Chromosome XIX was therefore finemapped and marker positions between $S$. viminalis and $P$. trichocarpa were compared to find evidence for translocations of chromosomal segments.

\section{MATERIALS AND METHODS}

\section{Plant material and phenotyping}

The $S_{3}$ mapping population (Berlin et al., 2010) is composed of full siblings from a cross between the male $S$. viminalis clone 81084 and the female S. viminalis clone 78195 . This cross has previously been demonstrated to produce individuals with variation in their resistance to the gall midge Dasineura marginemtorquens (Bremi) (Höglund et al., 2005). Crosses were made in 2000 and 2004 and the plants were reared in a greenhouse before planting in the field. The plants are maintained in a plant orchard in Pustnäs south of Uppsala, Sweden $\left(59.80^{\circ} \mathrm{N}, 17.67^{\circ} \mathrm{E}, 25 \mathrm{~m}\right.$ AOD). Gender determination was done twice in 2010 and in 2014

\section{QTL mapping}

QTL mapping was performed using phenotypic data from 282 individuals and a genome-wide linkage map presented in Berlin et al. (2010) where the $S_{3}$ linkage map for chromosome XV consists of two linkage groups (XV-1 and $\mathrm{XV}-2$; here called the old map). The analysis was made with the programme MapQTL 6.0 (Van Ooijen, 2009) using the non-parametric Kruskal-Wallis method and interval mapping with a regression model. The genome was scanned at $1 \mathrm{cM}$ intervals to determine putative QTLs associated with sex A significance threshold value, estimated as the logarithm of odds ratio (LOD), to determine significant QTLs was estimated with a permutation test of 1000 repetitions. A genome-wide threshold for a significant QTL was set at $P=0.05$. The two LOD confidence interval for each QTL were estimated using the LOD drop-off method (Lander and Botstein, 1989) based on the LOD-value at the peak position of the QTL. The markers closest to the 2-LOD region of the QTL were used to compare the lengths in cM of the QTL regions between the new and old linkage map. The proportion of the phenotypic variation explained by each significant QTL was estimated (\% Expl.). Maternal allelic effects were estimated as $A_{\mathrm{m}}=\left(\left(\mu_{\mathrm{ac}}+\mu_{\mathrm{ad}}\right)-\left(\mu_{\mathrm{bc}}+\mu_{\mathrm{bd}}\right)\right) / 2$, paternal allelic effects were estimated as $A_{\mathrm{p}}=\left(\left(\mu_{\mathrm{ac}}+\mu_{\mathrm{bc}}\right)-\left(\mu_{\mathrm{ad}}+\mu_{\mathrm{bd}}\right)\right) / 2$ and the allelic interaction effect were estimated as $A_{\mathrm{i}}=\left(\left(\mu_{\mathrm{ac}}+\mu_{\mathrm{bd}}\right)-\left(\mu_{\mathrm{ad}}+\mu_{\mathrm{bc}}\right)\right) / 2$ where $\mu_{\mathrm{ac}}, \mu_{\mathrm{bc}}, \mu_{\mathrm{ad}}$ and $\mu_{\mathrm{bd}}$ are the estimated phenotypic means of the four genotypic classes ac, bc, ad and bd obtained from an $\mathrm{ab} \times \mathrm{cd}$ cross (Van Ooijen, 2009).

\section{Finemapping}

With the aim of achieving a more precise localization of the sex determination QTL, new single-nucleotide polymorphism (SNP) markers with putative positions on the $S$. viminalis chromosome $\mathrm{XV}$ were genotyped in more individuals. Two hundred primer pairs were placed in exons spanning introns of approximately $500-1000$ base pairs on chromosome XV in the P. trichocarpe genome v. 1.1 (Tuskan et al., 2006). Genomic DNA was extracted from frozen young leaves with the FastDNA Kit (MP Biomedicals, Stockholm, Sweden). The gene fragments were amplified by PCR with DNA from each of the male and the female parent in a total volume of $15 \mu \mathrm{l}$. Each reaction contained $10 \mathrm{ng}$ genomic DNA, $1 \times$ PCR buffer II (Applied Biosystems, Stockholm, Sweden), $2.5 \mathrm{~mm} \mathrm{MgCl}_{2}$ (Applied Biosystems), $0.2 \mathrm{~mm}$ dNTP mix (Fermentas, Stockholm, Sweden), $0.5 \mu \mathrm{M}$ of each primer (Invitrogen, Stockholm, Sweden) and 0.5 U AmpliTaq Gold DNA polymerase (Applied Biosystems). The PCRs were run on a MyCycler thermal cycler (Bio-Rad Laboratories, Stockholm, Sweden) with a PCR profile consisting of $10 \mathrm{~min}$ denaturation at $95^{\circ} \mathrm{C}$ followed by 35 cycles of $30 \mathrm{~s}$ denaturation at $95^{\circ} \mathrm{C}, 30 \mathrm{~s}$ annealing at $58^{\circ} \mathrm{C}$ and $1 \mathrm{~min}$ extension at $72{ }^{\circ} \mathrm{C}$ with a final $10 \mathrm{~min} 72{ }^{\circ} \mathrm{C}$ step. Amplification success was determined by agarose gel electrophoresis. The PCR products were cleaned with $1 \mu \mathrm{l}$ of a mixture of Exonuclease I (New England BioLabs, Stockholm, Sweden) and Shrimp Alkaline Phosphatase (Fermentas) for every $5 \mu \mathrm{l}$ of PCR product and sequenced at Macrogen Inc. (Seoul, South Korea) on both strands using the forward and reverse PCR primers as sequencing primers. The chromatograms were visually inspected and edited using Lasergene SeqMan vs 9.1.0 (DNASTAR) and SNPs were identified. The new markers were named XV_f $x$, where $x$ is an arbitrary number and $f$ refer to 'finemapping'. In addition to these markers, two previously developed markers were genotyped: XV-5_sa and DT_6_sa 
(Berlin et al., 2010). SNPs were genotyped using the Illumina GoldenGate Assay at the SNP\&SEQ Technology Platform, Science for Life Laboratory at Uppsala University, Sweden, in 531 individuals, including the parental genotypes and the previously used 282 individuals (thus 247 new individuals were genotyped).

Markers were also developed evenly spaced on chromosome XIX in the $P$. trichocarpa genome. Primer pairs were designed and sequenced in both the male and the female parent in a similar manner as for the primers on chromosome XV and SNPs were genotyped together with the markers on chromosome XV. The new markers were named XIX_fx, adopting a similar nomenclature as for the markers on chromosome XV. In addition to the newly developed markers, eight previously developed markers were genotyped (Supplementary Table 1).

A new linkage map was constructed combining genotypes obtained with the old and the new markers using JoinMap 4.0 software (Van Ooijen, 2006) with a LOD of 4.0 for marker grouping. In addition to the genotypes, sex as a morphological marker was included in the linkage analyses. The mapping population was assigned as a cross pollinating population where markers could be polymorphic in one or both of the parents. The Kosambi mapping function and the Joinmap default settings was used for map construction. In case of more than one round of map building, we used the second round without allowing the programme to force any problematic markers into the map. Mendelian segregation of the different markers was tested with $\chi^{2}$ analysis. Maps were drawn using MapChart vs 2.2 (Voorrips, 2002). QTL mapping was conducted as described above using the new linkage map and phenotype data on sex from all 529 individuals in the mapping population.

Inferring the haplotypes in the sex determination QTL

In order to further narrow down the sex determination QTL, each individual's two haplotypes in the major QTL interval were inferred using the statistical software package PHASE v. 2.1 (Stephens et al., 2001; Stephens and Scheet, 2005). The analysis was restricted to the 10 markers surrounding the peak position of the QTL, that is, XV_f2, XV-5_sa, R_33_sa_pIII, R_51_sa, XV_f6, XV_f7, XV_f11, XV_f5, XV_f12 and XV_f21. PHASE adopts an iterative scheme to perform the inferences. Default values were used, which means 100 iterations, a thinning interval of 1 and a burn-in of 100. In order to obtain robust results the algorithm was applied five times with different seeds for the random number generator and consistency among runs was evaluated by examination of the haplotype frequency estimates. The inferred haplotypes were subsequently associated with the phenotypic data on sex.

\section{Comparative mapping and estimates of recombination rates}

SNP marker positions were determined in the Salix purpurea (Subgenus: Vetrix) genome assembly v 1.0, (DOE-JGI, http://phytozome.jgi.doe.gov/pz/ portal.html\#!linfo?alias=Org_Spurpurea), in the Salix suchowensis genome assembly (Dai et al., 2014) and in the P. trichocarpa genome assembly v. 3 (http://www.phytozome.net/poplar.php; Tuskan et al., 2006) using BLAST searches with the consensus DNA sequences from the male and the female parent (the marker sequences) containing the SNP markers on the S. viminalis chromosome XV. Similarly, marker positions were determined in the $P$. trichocarpa genome assembly for SNPs on the S. viminalis chromosome XIX. The positional information from the best BLAST hits was used to draw physical maps of chromosome XV in P. trichocarpa and S. purpurea and scaffolds of S. suchowensis that are part of chromosome XV and of chromosome XIX in P. trichocarpa. Also the positions of previously developed markers SCAR 354 (Genbank accession no. AY192565) and SCAR AE08 (Genbank accession no. AY192566) were examined by BLAST searches in the different genomes. In some instances, the best BLAST hit of the marker sequence was not on the homologous chromosome of $P$. trichocarpa and $S$. purpurea. In these cases, the position of the second best BLAST hit was used for the map construction.

The $S$. viminalis linkage map was aligned to the physical maps and the positions of homologous markers in the different species were shown by lines. The S. viminalis SD locus, that is, the interval with the markers XV-5_sa, R_33_sa_pIII and R_51_sa was analysed using the S. purpurea genome. Gene models located within the genomic interval were retrieved from the genome browser at http://phytozome.jgi.doe.gov/pz/portal.html\#!linfo?alias= Org_Spurpurea.

Recombination rates in base pairs per centimorgan were estimated between pairs of markers (a marker and its closest neighbour) across the $S$. viminalis genome. Only pairs of markers with a minimum of $0.1 \mathrm{cM}$ distance in the genetic map and 10 base pair distance in the physical map were used in the analysis. Physical positions of 309 SNPs distributed over the whole genome (Berlin et al., 2010) were obtained by BLAST searches against the S. purpurea genome as previously described. Genetic distances were taken from the linkage maps. The results were visualized in a histogram.

\section{RESULTS}

\section{QTL mapping with old linkage map}

Sex could be determined for 272 individuals out of the 282 that were genotyped, of which 137 were females and 135 were males. The analysis identified one highly significant QTL on chromosome XV associated with sex that explained $84.7 \%$ of the variation (Table 1). A large difference in the effect of female alleles was identified while this was not seen for the male alleles (Table 1). In the Kruskal-Wallis test, all markers on the two linkage groups on chromosome XV, except for the marker XV-5_sa, which was polymorphic only in the male, were significantly associated with sex. The markers R_33_sa_pIII and R_51_sa had particularly high significances $(K>200$; Table 2$)$.

\section{Finemapping and QTL mapping with new linkage map}

Two hundred primer pairs were positioned along the $P$. trichocarpa chromosome XV and were tested for amplification in both the female and the male parent. In all, 82 primer pairs resulted in successful amplification (41.0\%) and were directly sequenced. After inspection of the chromatograms, 40 SNPs were identified and selected for genotyping. Of the 39 SNPs that resulted in informative genotypes, 35 mapped to chromosome XV, 1 to chromosome VI and 3 to chromosome XII (Supplementary Table 1). When performing BLAST searches with the marker sequences containing the SNP markers that mapped to VI and XII to the P. trichocarpa genome, the best BLAST hits were also on VI and XII (Supplementary Table 1), suggesting that the intended gene fragments had not been amplified. The gene fragments that mapped to chromosome XII are likely the paralogous

Table 1 Results from the QTL analysis using the old and new maps

\begin{tabular}{|c|c|c|c|c|c|c|c|c|c|c|c|}
\hline Chr. & $\mathrm{N}$ & Marker ${ }^{a}$ & Pos. (cM) & $\angle O D$ & $\angle O D$ thresh. $^{\text {b }}$ & Var. expl. $(\%)^{c}$ & Female alleles & Male alleles & Interaction & QTL interval $(C M)^{d}$ & QTL-marker interval (cM) \\
\hline XV (old) & 282 & R_51_sa & 16.7 & 111.1 & 4.1 & 84.7 & -0.988 & -0.007 & -0.016 & 6.2 & 45.6 \\
\hline IX_2 (new) & 529 & IX_16_sa & 44.4 & 4.6 & 4.1 & 4.0 & -0.091 & -0.257 & 0.138 & 20.8 & 23.8 \\
\hline XV (new) & 529 & sex & 39.3 & 439.2 & 4.1 & 97.9 & 1.000 & 0.004 & 0.004 & 0.2 & 1.8 \\
\hline
\end{tabular}

Abbreviations: Chr., chromosome; Pos., position; thresh., threshold.

a Marker closest to the peak position of the QTL.

bGenome-wide LOD threshold at 0.05 level.

cPhenotypic variation explained by the QTL.

dTwo-LOD QTL interval.

e Region between markers closest to the position on each side of the LOD peak position representing a drop of two LODs. 
Table 2 Results from the Kruskal-Wallis analysis showing all markers on the old map of chromosome XV (LG 1 and 2)

\begin{tabular}{|c|c|c|c|c|c|c|c|c|c|c|c|}
\hline \multirow[t]{2}{*}{ Position, cM (LG) } & \multirow[t]{2}{*}{ Marker } & \multirow[t]{2}{*}{ K } & \multirow[t]{2}{*}{$\mathrm{P}$ value } & \multirow[t]{2}{*}{$\mathrm{N}$} & \multicolumn{3}{|c|}{ Mean of genotype } & \multicolumn{2}{|c|}{ Mean of genotype } & \multicolumn{2}{|c|}{ Mean of genotype } \\
\hline & & & & & $h h$ & $h k$ & $k k$ & II & Im & $n n$ & $n p$ \\
\hline $4.894(1)$ & WPMS5 & 18.862 & $1 \times 10^{-7}$ & 75 & 1.210 & 1.454 & 1.869 & & & & \\
\hline $0.000(2)$ & DT_6_sa & 122.301 & $1 \times 10^{-7}$ & 261 & & & & 1.160 & 1.846 & & \\
\hline $14.744(2)$ & R_33_sa_pllI & 229.151 & $1 \times 10^{-7}$ & 261 & & & & 1.023 & 1.962 & & \\
\hline $53.582(2)$ & R_32_sa_pl & 13.383 & $1 \times 10^{-6}$ & 261 & & & & 1.604 & 1.376 & & \\
\hline
\end{tabular}

Abbreviations: K, the Kruskal-Wallis test statistic based on ranks; LG, linkage groups; NS, not significant.

\section{S. suchowensis}

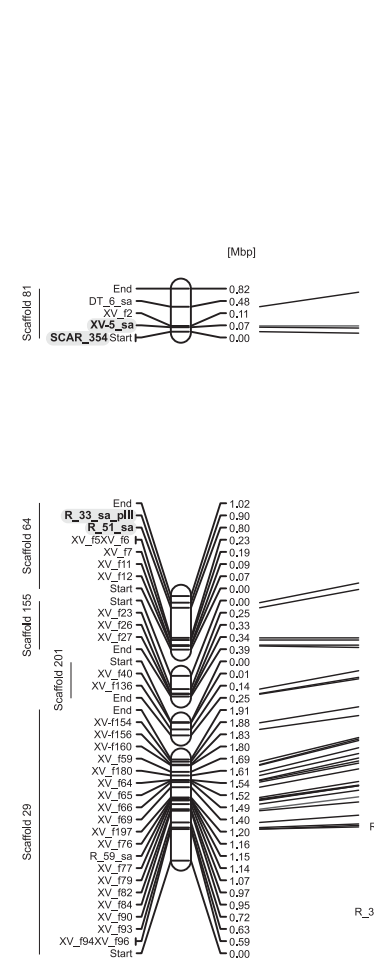

P. trichocarpa

Chr. XV
S. purpurea

Chr. XV

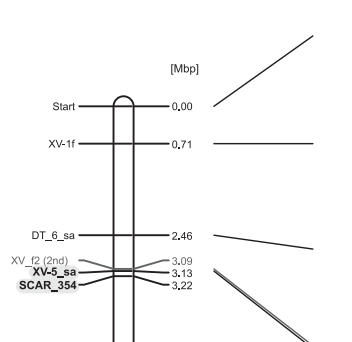

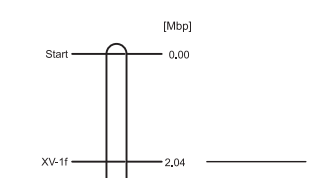

S. viminalis

Chr. XV

QTL on Chr. XV

in S. viminalis 


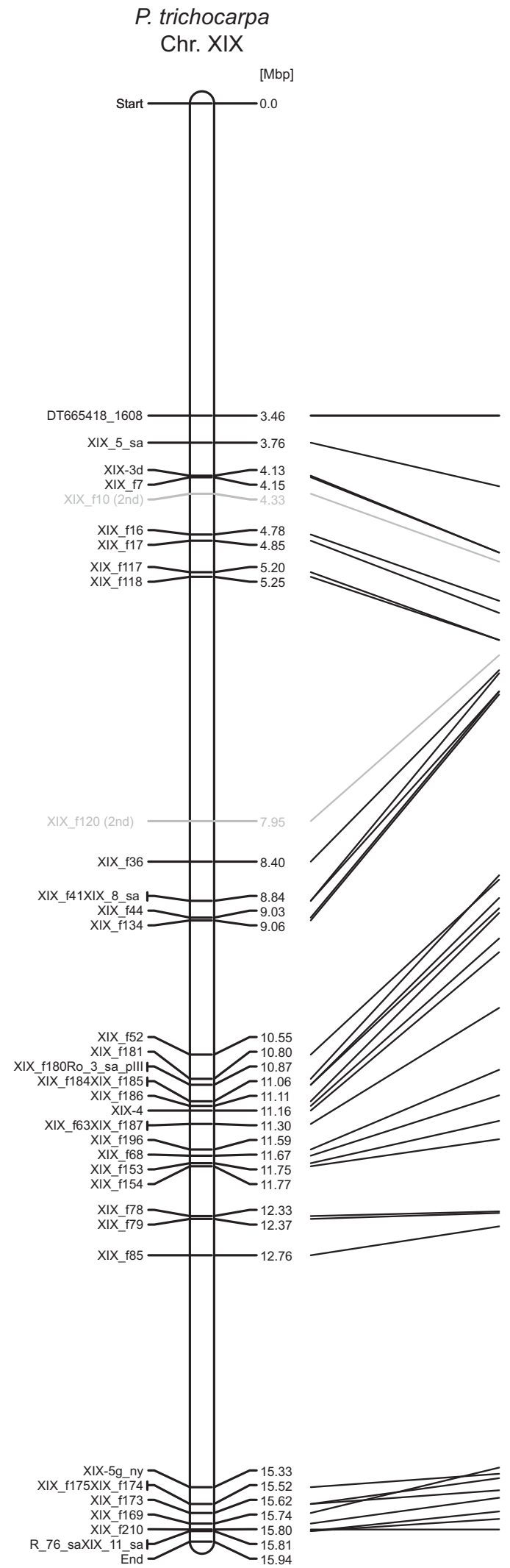

\author{
S. viminalis \\ Chr. XIX \\ [cM]
}
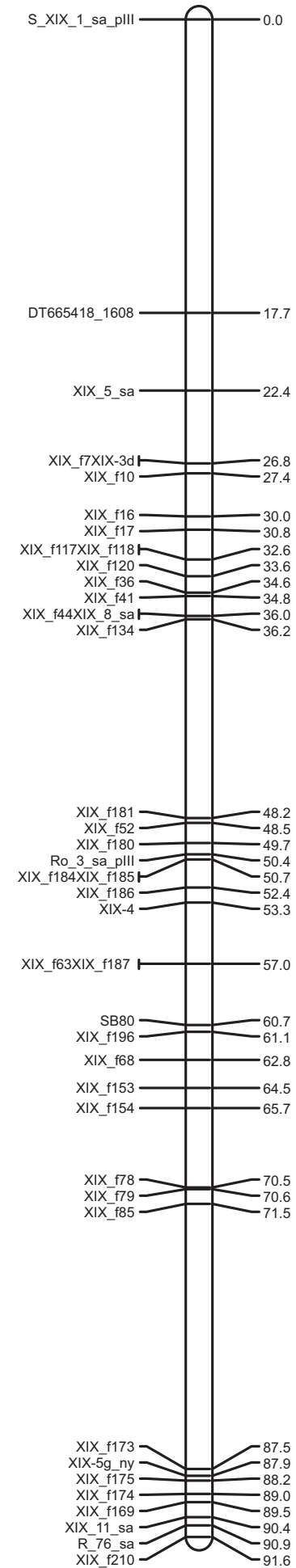

Figure $2 S$. viminalis and $P$. trichocarpa chromosome XIX. The $P$. trichocarpa map shows the positions of the markers that were determined by BLAST searches of the $S$. viminalis marker sequences to the $P$. trichocarpa genome v. 3. The $S$. viminalis map represents the new linkage map of chromosome XIX. Homologous markers between the $P$. trichocarpa and the $S$. viminalis maps were connected by lines. For some markers, the best BLAST hits in the $P$. trichocarpa genome were not on chromosome XIX. In these cases, the second best hits were used, which is labelled also in the figure. 
gene fragments to those on chromosome $\mathrm{XV}$, as large parts of chromosome XII and XV contain paralogous regions that originate from the whole-genome duplication (that is, the Salicoid event) that took place before the divergence of poplars and willows (Tuskan et al., 2006). Fourteen of the new markers that mapped were polymorphic in both parental genotypes (2:1:1 segregation), nine in the maternal parent and twelve in the paternal parent only (3:1 segregation; Supplementary Table 1). The morphological marker 'sex' mapped close to XV-5_sa on chromosome XV (Figure 1).

A total of 210 primer pairs were developed on $P$. trichocarpa chromosome XIX, of which 96 resulted in successful amplification $(45.7 \%)$ that were directly sequenced. Forty-two SNPs were identified and genotyped. Of the 36 SNPs that resulted in informative genotypes, 31 mapped to chromosome XIX, 1 to chromosome VII, 2 to chromosome XIII, 1 to chromosome XVIII and 1 did not map. BLAST searches with the marker sequences containing the SNP markers that mapped to chromosome XIII and XVIII to the $P$. trichocarpa genome, showed that the best BLAST hits were also on XIII and XVIII (Supplementary Table 1), suggesting that the intended gene fragments were not amplified. In the case of the marker sequences on chromosome XIII, the paralogous gene fragments could have been amplified as XIII and XIX contain paralogous regions that originate from the Salicoid event (Tuskan et al., 2006). However, the best BLAST hit of the marker sequence containing the marker that mapped to chromosome VII was on chromosome XIII, suggesting that again the paralogue had been amplified. However, the fact that it mapped to chromosome VII in S. viminalis could indicate that some rearrangement has happened between chromosome VII and XIII since the split between $S$. viminalis and $P$. trichocarpa. Eight of the new markers that mapped were polymorphic in both parental genotypes, thirteen in the maternal parent and fourteen in the paternal parent only (Supplementary Table 1).

Sex could be determined for 523 individuals out of the 529 that were genotyped. Of these, 271 carried catkins with female flowers and were thus scored as females and 252 carried catkins with male flowers and were scored males. This resulted in a slightly female biased sex ratio (1:0.93).

QTL mapping revealed, as in the previous analysis, a highly significant QTL for sex on chromosome XV, which explained as much as $97.9 \%$ of the variation (Table 1 ). The peak position was at the morphological marker 'sex' very close to the marker XV-5_sa and the tightly linked markers R_33_sa_pIII and R_51_sa. The difference in the effects of the female alleles was 1, which means that one of the alleles was completely associated with sex as the male sex was scored as 1 and the female sex as 2. The 2-LOD region connected to the closest markers was significantly reduced compared with the analysis with the old map, because of the much tighter linkage map (Table 1). The nonparametric Kruskal-Wallis analysis also supported a strong signal in this region as nine markers had exceptionally high $K$ values $(K>200$; Table 3). The markers XV-5_sa and XV_f5 were, however, nonsignificant as they were only polymorphic in the males (Table 3). In addition to the QTL on chromosome XV, there was also a significant QTL on chromosome IX that explained 4\% of the variation of the sex phenotype. For this QTL, the difference between the male alleles was higher compared with between female alleles.

\section{Haplotype inference and analyses of phenotypic effects}

By applying a statistical threshold of $P<0.1$, haplotypes were inferred in 502 individuals ( 259 females and 243 males) for 10 markers in the QTL on chromosome XV (XV_f2, XV-5_sa, R_33_sa_pIII, R_51_sa, XV_f6, XV_f7, XV_f11, XV_f5, XV_f12 and XV_f21). Four common haplotypes were found, ranging between 23 and $25 \%$ in frequency, representing the non-recombinant parental haplotypes. Recombination was marginally more common in the maternal parent compared with the paternal parent as 32 or $6.4 \%$ of the haplotypes with a maternal origin and 26 or $5.2 \%$ of the haplotypes with a paternal origin were recombinant haplotypes. However, a larger number of different recombinant haplotypes were formed in the maternal parent compared with the paternal parent as among the recombinant haplotypes with a maternal origin there were 10 different haplotypes, and among the recombinant haplotypes with a paternal origin there were only 5 different haplotypes (Table 4 ). When associating these haplotypes to sex, an interesting pattern emerged. The haplotypes with a paternal origin were to the same degree found in male and female offspring (Table 4). On the other hand, the haplotypes with a maternal origin were found either in male or female offspring (Table 4). The Maternal-1 parental haplotype was female specific and as a consequence the Maternal-2 parental haplotype was male specific (Table 4). A similar pattern was also found for the recombinant haplotypes, where Maternal-3 to Maternal-7 were female specific and Maternal- 8 to Maternal-12 were male specific (Table 4). This agrees with results from the QTL mapping that showed large differences between maternal alleles and a complete association of one allele with sex (Table 1). Common to all female-specific haplotypes were the

Table 3 Results from the Kruskal-Wallis analysis showing the most significant markers on the new map of chromosome XV

\begin{tabular}{|c|c|c|c|c|c|c|c|c|c|c|c|}
\hline \multirow[t]{2}{*}{ Position, cM } & \multirow[t]{2}{*}{ Marker } & \multirow[t]{2}{*}{$\mathrm{K}$} & \multirow[t]{2}{*}{$\mathrm{P}$ value } & \multirow[t]{2}{*}{$\mathrm{N}$} & \multicolumn{3}{|c|}{ Mean of genotype } & \multicolumn{2}{|c|}{ Mean of genotype } & \multicolumn{2}{|c|}{ Mean of genotype } \\
\hline & & & & & $h h$ & $h k$ & $k k$ & /I & Im & $n n$ & $n p$ \\
\hline 24.1 & DT_6_sa & 249.6 & $1 \times 10^{-7}$ & 515 & & & & 1.159 & 1.856 & & \\
\hline 37.8 & $X V_{-} f 2$ & 483.5 & $1 \times 10^{-7}$ & 512 & & & & 1.008 & 1.981 & & \\
\hline 39.3 & Sex & 511.0 & $1 \times 10^{-7}$ & 516 & & & & 1.004 & 2.000 & & \\
\hline 39.7 & XV-5_sa & 0.168 & NS & 516 & & & & & & 1.524 & 1.506 \\
\hline 39.8 & R_51_sa & 262.0 & $1 \times 10^{-7}$ & 267 & & & & 2.000 & 1.008 & & \\
\hline 39.8 & R_33_sa_pllI & 262.0 & $1 \times 10^{-7}$ & 267 & & & & 1.008 & 2.000 & & \\
\hline 42.8 & $X V \_f 6$ & 221.0 & $1 \times 10^{-7}$ & 515 & 1.983 & 1.541 & 1.058 & & & & \\
\hline 43.2 & $X V \_f 7$ & 215.0 & $1 \times 10^{-7}$ & 514 & 1.059 & 1.539 & 1.975 & & & & \\
\hline 43.5 & XV_f11 & 431.6 & $1 \times 10^{-7}$ & 513 & & & & 1.966 & 1.048 & & \\
\hline 43.5 & XV_f5 & 0.006 & NS & 515 & & & & & & 1.516 & 1.513 \\
\hline 44.3 & $X V \_f 12$ & 204.2 & $1 \times 10^{-7}$ & 515 & 1.967 & 1.537 & 1.074 & & & & \\
\hline
\end{tabular}

Abbreviations: $K$, the Kruskal-Wallis test statistic based on ranks; NS, not significant. 
Table 4 Haplotype inferences in the major QTL and associations between haplotypes and sex

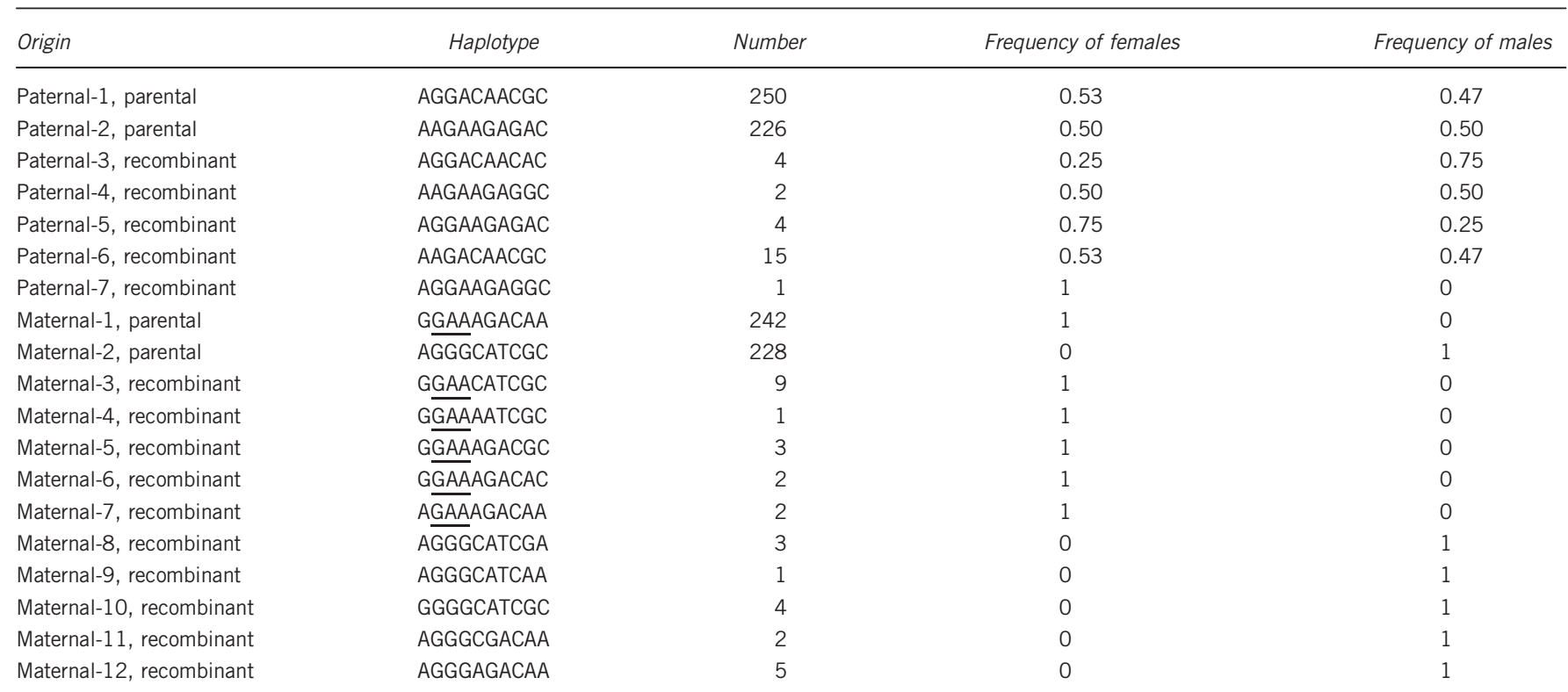

Sex was recorded in 523 plants where 271 carried catkins with female flowers and 252 carried catkins with male flowers. By applying a statistical threshold of $P<0.10$, haplotypes were inferred in 502 individuals (259 females and 243 males). The female-specific haplotype at the SD locus was GAA at the markers XV_5_sa, R_33_sa_plII and R_51_sa (underlined).

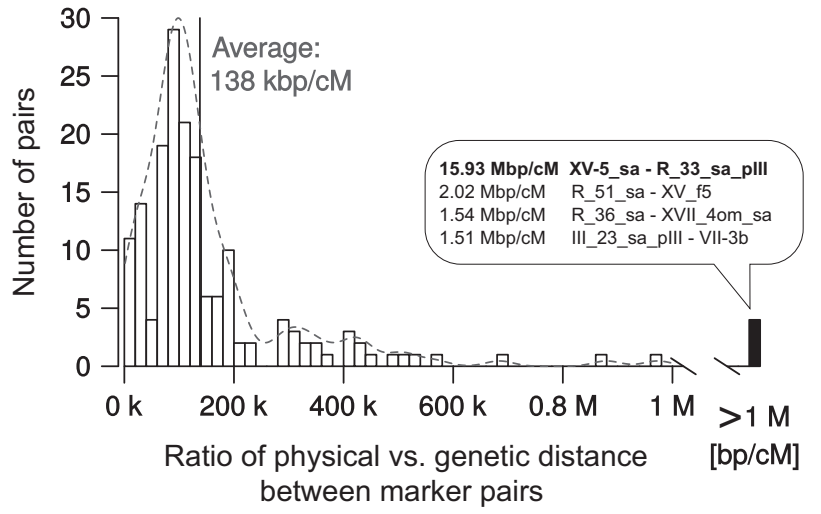

Figure 3 Distribution of pairwise recombination rates in base pairs per cM between pairs of markers. Physical distances were obtained from the $S$. purpurea 1.0 genome and genetic distances from the new linkage map of S. viminalis. The lowest recombination rate was found in the SD locus, that is, between markers XV-5_sa and R_33_sa_PIII with $15.93 \times 10^{6}$ base pairs per $\mathrm{CM}$.

combination of $\mathrm{G}, \mathrm{A}$ and $\mathrm{A}$ at the XV-5_sa, R_33_sa_pIII and R_51_sa markers. These three markers are referred to as the SD locus (Table 4).

\section{Comparative mapping}

When comparing the $S$. viminalis linkage map to the physical maps of S. purpurea, S. suchowensis and P. trichocarpa for chromosome XV, the synteny is overall shared, and no major rearrangement were detected (Figure 1). Individual marker sequences had a marginally better hit outside chromosome XV in S. purpurea and P. trichocarpa, that is, for $P$. trichocarpa the markers XV_f2 and XV_f82 had the best BLAST hit on chromosome XII, which is known to contain paralogous regions to chromosome XV. In S. purpurea, the markers XV_f51 and XV_f93 mapped best to two different scaffolds (scaffold 4340 and 0911 respectively). In all cases, the second best BLAST hit was of nearly identical quality and the position of that second hit was in syntheny. The three markers in the $S$. viminalis SD locus (XV-5_sa, R_33_sa_pIII and R_51) were present in all species, however, in S. suchowensis, XV-5_sa was on a different scaffold than the other two markers. SCAR 354 was located close to XV-5_sa on chromosome XV in the genomes of S. suchowensis and P. trichocarpa (Figure 1), but in the genome of $S$. purpurea it was located on scaffold 2584. SCAR AE08 did not map to chromosome XV in any of the species, but poor matches were found on chromosome V in P. trichocarpa, chromosome XVII in S. purpurea and to scaffold 238 in S. suchowensis. When comparing the $S$. viminalis linkage map to the physical maps of $P$. trichocarpa for chromosome XIX, again the overall synteny was shared and no chromosome XV marker mapped to XIX or vice versa (Figure 2). Marker XIX_f10, however, had a slightly better hit on scaffold 75, whereas marker XIX_f120 mapped marginally better to scaffold 53 (Figure 2). The SD locus, that is, between the markers XV-5_sa and R_51_sa represents a genomic region of about $2.5 \mathrm{Mbp}$ in the $S$. purpurea genome, which harbours 48 protein-coding genes (Supplementary Table 2). When estimating the recombination rates between pairs of markers, the mean ratio for 171 analysed pairs was $138 \mathrm{kbp}$ per cM (Figure 3). The most extreme ratio in the whole genome was found between the markers XV-5_sa and R_51_sa where the ratio was approximately $16 \mathrm{Mbp}$ per $\mathrm{cM}$ (Figure 3 ), which is 115 times lower compared with the genome average.

\section{DISCUSSION}

In this study, we have for the first time identified and positioned one single SD locus on chromosome XV in the basket willow, S. viminalis. As the SCAR 354 marker that previously was demonstrated to be sex linked in other S. viminalis accessions (Alström-Rapaport et al., 1998; Gunter et al., 2003a) appear to be positioned near the XV-5_sa marker in the SD locus (based on BLAST searches in the genomes of S. suchowensis and P. trichocarpa), our and previous results together supports a general sex determination mechanism in $S$. viminalis 
involving the SD locus in a so far unknown way. As the morphological trait 'sex' mapped near the XV-5_sa marker, this further strengthen the close association between the SD locus and sex as a morphological trait in this species. We further demonstrated that all female offspring had one maternally inherited haplotype, supporting a female heterogametic system. Sex determination could then either be under the control of a dominant $\mathrm{W}$-like haplotype or alternatively, $\mathrm{Z}$ dosage determines sex. These two mechanisms are fundamentally different as in the first case the dominant W-like haplotype is assumed to harbour a master gene controlling female development, which is equivalent to the sex determination system in mammals (the SRY master male determining gene located on the $\mathrm{Y}$ chromosome). In the other case, sex would be determined by the dosage of one or more Z-linked genes and a higher dose would thus be required for male development, which would resemble the sex determination mechanism found in birds (Nanda et al., 2008; Smith et al., 2009). Further work is, however, needed in order to distinguish between these two mechanisms.

With the phase analysis, we reduced the SD locus to three markers that corresponded to a $2.5-\mathrm{Mbp}$ genomic region in the $S$. purpurea genome, a region that harbours 48 protein-coding genes. This value should, however, be seen as an approximation and not as a definitive size of the region as $S$. viminalis and $S$. purpurea might have undergone lineage-specific changes since they diverged. In addition, if the sex determination regions are the same in S. purpurea and S. viminalis, the $\mathrm{Z}$ - and $\mathrm{W}$-like genomic regions might have started to differentiate. As the $S$. pupurea genome assembly is based on sequencing data from a female individual the assembly process for this region might be a major challenge. There are reasons to believe that there are problems with the assembly as the SCAR 354 marker mapped to a scaffold (quite likely that it should be in the SD locus near XV-5_sa also in S. purpurea), and also that $S$. purpurea chromosome XV is much longer than chromosome XV in poplar. This can obviously be a problem when using the $S$. purpurea genome to get physical distances between the markers. If the region is misassembled and by that too long, the measure base pairs per cM will be overestimated in this region compared with the rest of the genome. As we found that the SD locus had an extremely low recombination rate compared with the rest of the genome this could then either be a consequence of misassembly or reflect suppression of recombination in the SD locus, or both. However, low recombination was also found when $P$. trichocarpa was used to estimate the physical distances between the markers (data not shown) indicating that the effect is not caused by potential problems in the $S$. purpurea genome assembly alone. In a female heterogametic system, recombination should be supressed only in females but not in males. However, in the present study, it was not possible to make this comparison, as only one of the three markers in the SD locus was polymorphic in the male parent (that is, XV-5_sa). Suppressed recombination in the SD locus could be a consequence of the position of the locus near the centromere, where recombination rates are known to be reduced. In other species, SD loci are located in pericentromeric regions, for example, in $P$. tremuloides (Kersten et al., 2014), P. alba (Paolucci et al., 2010), papaya (Yu et al., 2007) and asparagus (Telgmann-Rauber et al., 2007). However, as the recombination rate in the $\mathrm{SD}$ locus in the present study is the lowest found in the whole genome, other mechanisms that suppress recombination are probably operating in addition to the mere localization near the centromere on chromosome XV.

As both willows and poplars are dioecious, the most parsimonious explanation is that dioecy evolved before the two lineages diverged more than 45 million years ago. How come heteromorphic sex chromosomes have not evolved during this time? One likely explanation is that there has been high rates of sex chromosome turnover in the Salicaceae family, which previous studies support. For example, SD loci in poplars are located on different positions on chromosome XIX and both male and female heterogamety is found. In contrast, S. viminalis is female heterogametic and the SD locus is located on chromosome XV. Although the SD loci have different positions in poplar and willow genomes, they could still be homologous and harbouring homologous sex determination genes, but through translocations have swapped genomic positions. If we hypothesize that markers are expected to have the same position in the S. viminalis and the $P$. trichocarpa genomes, differences can be the result of lineagespecific rearrangements, such as translocations, inversions, fusions and fissions. Using this strategy, we previously demonstrated that a number of both large and small rearrangements have happened as the split between poplars and willows, for example, the fusion in the willow lineage between parts of poplar chromosome I and poplar chromosome XVI, and the fission of poplar chromosome I (Berlin et al., 2010). The resolution of this method depends on the density of the marker in the genomic region of interest and the closer the distances between the markers the smaller rearrangements can be detected. By adopting this method here on the finemapped chromosome XV and XIX, we conclude that no chromosome XIX marker mapped to chromosome XV, or vice versa. Thus, the SD locus in $S$. viminalis is likely not related to the SD locus on chromosome XIX in $P$. trichocarpa, which suggests that different sex determination mechanisms exist in willows and poplars. Therefore, willows and poplars together represent a system with high sex chromosome turn over rates, where new master sex determination genes are replacing the old ones on new chromosomes. This rapid turnover has also led to repeated transitions between male and female heterogamety in this plant family. Why is the turnover rate high in the Salicaceae family? It has been demonstrated that sexually antagonistic selection can result in the spread of new sex determination genes when linked to another autosomal gene and this would be particularly likely in systems with undifferentiated sex chromosomes (van Doorn and Kirkpatrick, 2007). In another theoretical study, it was also shown that sexually antagonistic selection can cause transitions between male and female heterogamety (van Doorn and Kirkpatrick, 2010). More studies are needed to investigate the evolutionary processes responsible for the high rates of turnover in sex determination mechanisms in the Salicaceae plant family.

\section{DATA ARCHIVING}

Primers used for SNP identification are included in the Supplementary Table 1. The linkage map, the genotype data and the phenotype data have been deposited on Dryad at http://datadryad.org under doi:10.5061/dryad.1qh3k.

\section{CONFLICT OF INTEREST}

The authors declare no conflict of interest.

\section{ACKNOWLEDGEMENTS}

We thank Luisa Ghelardini for assistance in the phenotyping in the field and Urban Pettersson for performing the crossings. We also thank Yvonne Tillman for doing the DNA extractions. Genotyping was performed by the SNP\&SEQ Technology Platform in Uppsala. The platform is part of Science for Life Laboratory at Uppsala University and supported as a national infrastructure by the Swedish Research Council. The Salix purpurea sequence data were produced by the US Department of Energy Joint Genome Institute. The research was supported by the Swedish Research Council. We are very grateful 
to the three anonymous referees who gave insightful comments and suggestions that greatly improved the manuscript.

Alström-Rapaport C, Lascoux M, Gullberg U (1997). Sex determination and sex ratio in the dioecious shrub Salix viminalis L. Theor Appl Genet 94: 493-497.

Alström-Rapaport C, Lascoux M, Wang YC, Roberts G, Tuskan GA (1998). Identification of a RAPD marker linked to sex determination in the basket willow (Salix viminalis L.). J Hered 89: 44-49.

Bachtrog D (2008). The temporal dynamics of processes underlying $Y$ chromosome degeneration. Genetics 179: 1513-1525.

Berlin S, Ellegren H (2004). Chicken W: a genetically uniform chromosome in a highly variable genome. Proc Natl Acad Sci USA 101: 15967-15969.

Berlin S, Lagercrantz U, von Arnold S, Öst T, Rönnberg-Wästljung AC (2010). High-density linkage mapping and evolution of paralogs and orthologs in Salix and Populus. BMC Genomics 11: 129.

Boucher LD, Manchester SR, Judd WS (2003). An extinct genus of Salicaceae based on twigs with attached flowers fruits, and foliage from the Eocene Green River Formation of Utah and Colorado, USA. Am J Bot 90: 1389-1399.

Charlesworth B, Charlesworth D (2000). The degeneration of Y chromosomes. Philos Trans $R$ Soc Lond B 355: 1563-1572.

Charlesworth D (2002). Plant sex determination and sex chromosomes. Heredity 88 : 94-101.

Charlesworth D, Charlesworth B, Marais G (2005). Steps in the evolution of heteromorphic sex chromosomes. Heredity 95: 118-128.

Charlesworth D, Mank JE (2010). The birds and the bees and the flowers and the trees: lessons from genetic mapping of sex determination in plants and animals. Genetics 186: 9-31.

Dai X, Hu Q, Cai Q, Feng K, Ye N, Tuskan GA et al. (2014). The willow genome and divergent evolution from poplar after the common genome duplication. Cell Res 24: 1274-1277.

Gaudet M, Jorge V, Paolucci I, Beritognolo I, Mugnozza GS, Sabatti M (2008). Genetic linkage maps of Populus nigra L. including AFLPs, SSRs, SNPs, and sex trait. Tree Genet Genomes 4: 25-36.

Gunter LE, Roberts GT, Lee K, Larimer FW, Tuskan GA (2003a). The development of two flanking SCAR markers linked to a sex determination locus in Salix viminalis L. J Hered 94: 185-189.

Gunter LE, Kopp RF, McCord RP, Tuskan GA (2003b). Analysis of sex-linked, sequencecharacterized amplified region markers in Salix eriocephala. Can J Forest Res 33: 1785-1790.

Hillier LW, Miller W, Birney E, Warren W, Hardison RC, Ponting CP et al. (2004). Sequence and comparative analysis of the chicken genome provide unique perspectives on vertebrate evolution. Nature 432: 695-716.

Höglund S, Larsson S, Wingsle G (2005). Both hypersensitive and non-hypersensitive responses are associated with resistance in Salix viminalis against the gall midge Dasineura marginemtorquens. J Exp Bot 56: 3215-3222.

Kersten B, Pakull B, Fladung M (2012). Mapping of the sex trait and sequence analysis of two sex linked genomic regions in Populus tremuloides. ScienceMed $\mathbf{3}$ : 203-210.

Kersten B, Pakull B, Groppe K, Lueneburg J, Fladung M (2014). The sex-linked region in Populus tremuloides Turesson 141 corresponds to a pericentromeric region of about two million base pairs on $P$. trichocarpa chromosome 19. Plant Biol 16: 411-418.

Lander ES, Botstein D (1989). Mapping mendelian factors underlying quantitative traits using RFLP linkage maps. Genetics 121: 185-199.

Manchester SR, Judd WS, Handley B (2006). Foliage and fruits of early poplars (Salicaceae: Populus) from the eocene of Utah, Colorado, and Wyoming. Int J Plant Sci 167: 897-908.

Mank JE, Avise JC (2009). Evolutionary diversity and turn-over of sex determination in teleost fishes. Sex Dev 3: 60-67.

Miura I (2007). An evolutionary witness: the frog Rana rugosa underwent change of heterogametic sex from XY male to ZW female. Sex Dev 1: 323-331.

Moghadam HK, Pointer MA, Wright AE, Berlin S, Mank JE (2012). W chromosome expression responds to female-specific selection. Proc Natl Acad Sci USA 109: 8207-8211.
Nanda I, Schlegelmilch K, Haaf T, Schartl M, Schmid M (2008). Synteny conservation of the $Z$ chromosome in 14 avian species ( 11 families) supports a role for $Z$ dosage in avian sex determination. Cytogenet Genome Res 122: 150-156.

Pakull B, Groppe K, Meyer M, Markussen T, Fladung M (2009). Genetic linkage mapping in aspen (Populus tremula L. and Populus tremuloides Michx.). Tree Genet Genomes 5: 505-515.

Paolucci I, Gaudet M, Jorge V, Beritognolo I, Terzoli S, Kuzminsky E et al. (2010). Genetic linkage maps of Populus alba L. and comparative mapping analysis of sex determination across Populus species. Tree Genet Genomes 6: 863-875.

Perrin N (2009). Sex reversal: a fountain of youth for sex chromosomes? Evolution 63 : 3043-3049.

Renner SS (2014). The relative and absolute frequencies of angiosperm sexual systems: Dioecy, monoecy, gynodioecy, and an updated online database. Am J Bot 101: 1588-1596.

Semerikov V, Lagercrantz U, Tsarouhas V, Rönnberg-Wästljung A, Alström-Rapaport C, Lascoux M (2003). Genetic mapping of sex-linked markers in Salix viminalis L. Heredity 91: 293-299.

Slancarova V, Zdanska J, Janousek B, Talianova M, Zschach C, Zluvova J et al. (2013). Evolution of sex determination systems with heterogametic males and females in Silene. Evolution 67: 3669-3677.

Smith CA, Roeszler KN, Ohnesorg T, Cummins DM, Farlie PG, Doran TJ et al. (2009). The avian Z-linked gene DMRT1 is required for male sex determination in the chicken. Nature 461: 267-271.

Stephens M, Scheet P (2005). Accounting for decay of linkage disequilibrium in haplotype inference and missing data imputation. Am J Hum Genet 76: 449-462.

Stephens M, Smith NJ, Donnelly P (2001). A new statistical method for haplotype reconstruction from population data. Am J Hum Genet 68: 978-989.

Telgmann-Rauber A, Jamsari A, Kinney MS, Pires JC, Jung C (2007). Genetic and physical maps around the sex-determining M-locus of the dioecious plant asparagus. Mol Genet Genomics 278: 221-234.

Temmel NA, Hardeep SR, Cronk QCB (2007). Sequence characterization of the putatively sex-linked Ssu 72-like locus in willow and its homologue in poplar. Can J Bot 85: 1092-1097.

Tuskan GA, DiFazio S, Faivre-Rampant P, Gaudet M, Harfouche A, Jorge V et al. (2012). The obscure events contributing to the evolution of an incipient sex chromosome in Populus: a retrospective working hypothesis. Tree Genet Genomes 8: 559-571.

Tuskan GA, Difazio S, Jansson S, Bohlmann J, Grigoriev I, Hellsten U et al. (2006). The genome of black cottonwood, Populus trichocarpa (Torr. \& Gray). Science 313: 1596-1604.

van Doorn GS, Kirkpatrick M (2007). Turnover of sex chromosomes induced by sexual conflict. Nature 449: 909-912.

van Doorn GS, Kirkpatrick M (2010). Transitions between male and female heterogamety caused by sex-antagonistic selection. Genetics 186: 629-U294.

Van Ooijen JW (2006). JoinMap ® 4, Software for the Calculation of Genetic Linkage Maps in Experimental Populations. Kyazma BV (ed), Wageningen, Netherlands.

Van Ooijen JW. (2009). MapQTL ® 6, Software for the Mapping of Quantitative Trait Loci in Experimental Populations. Kyazma BV (ed), Wageningen, Netherlands.

Voorrips RE (2002). MapChart: software for the graphical presentation of linkage maps and QTLs. J Hered 93: 77-78.

Yin T, Difazio SP, Gunter LE, Zhang X, Sewell MM, Woolbright SA et al. (2008). Genome structure and emerging evidence of an incipient sex chromosome in Populus. Genome Res 18: 422-430.

Yu Q, Hou S, Hobza R, Feltus FA, Wang X, Jin W et al. (2007). Chromosomal location and gene paucity of the male specific region on papaya $Y$ chromosome. Mol Genet Genomics 278: 177-185.

(c) (i) (5) $\odot$ This work is licensed under a Creative Commons Attribution-NonCommercial-NoDerivs 3.0 Unported License. The images or other third party material in this article are included in the article's Creative Commons license, unless indicated otherwise in the credit line; if the material is not included under the Creative Commons license, users will need to obtain permission from the license holder to reproduce the material. To view a copy of this license, visit http://creativecommons.org/licenses/by-nc-nd/3.0/

Supplementary Information accompanies this paper on Heredity website (http://www.nature.com/hdy) 